\title{
Tipping the balance in autoimmune disease Mary Collins
}

Address: Wyeth Research, Cambridge Park Drive, Cambridge, MA 02140, USA.

Published: 2 November 2007

Genome Biology 2007, 8:317 (doi:10.1 186/gb-2007-8-10-317)

The electronic version of this article is the complete one and can be found online at http://genomebiology.com/2007/8/10/317

(c) 2007 BioMed Central Ltd

A report on the FASEB Summer Research Conference on Autoimmunity, Saxtons River, USA, I4-29 July 2007.

We have long known that there are both genetic and nongenetic components in autoimmune disease. Although underlying genetic contributions can increase the likelihood of developing autoimmune disease, they do not ensure it, implying that environmental components also affect the outcome. The major themes for this year's FASEB conference on autoimmunity were the emerging data on genetic polymorphisms linked with human autoimmune disease, and recent developments in our current understanding of immune mechanisms implicated in autoimmune disease. This report focuses on how we can begin to integrate the new genetic data into our current understanding of normal and pathological immune processes.

\section{Genome-wide linkage analysis}

As pointed out by Joan Goverman (University of Washington, Seattle, USA), we now know that human autoimmune diseases are truly diseases of immune dysfunction. The proof of this lies in the genes that have recently been associated with the development of multiple sclerosis (MS), type 1 diabetes, systemic lupus erythematosis (SLE), Crohn's disease (CD) and rheumatoid arthritis (RA). Interestingly, some of the predisposing genes are shared between different diseases, suggesting common mechanisms underlying the pathology of these diseases. There are also unique genetic components, however, indicating that distinct pathways are contributing to the etiology of each disease.

Large groups of researchers have now come together to share populations of patients and identify genetic linkages using large-scale analysis of single-nucleotide polymorphisms (SNPs). The power of this approach is that it is hypothesisneutral, and large datasets allow the detection of the weaker genetic linkages found for complex multigenic traits. David
Hafler (Harvard Medical School, Boston, USA) previewed data from the International Multiple Sclerosis Genetics Consortium. In this study, 931 MS patients and their parents were evaluated for SNPs using DNA microarrays. Additional MS families and healthy controls were tested for replication of the findings, and the likelihood of association of a given SNP polymorphism with MS was determined. As expected, the HLA locus had the largest association with MS, underscoring the point that the immune system contributes to disease predisposition. The exciting news was that additional genes of immune function are also associated with MS. The most significant linkages were seen with genes for the alpha chain of the IL2 receptor (IL2RA) and the receptor for IL7. The cytokines IL2 and IL7 are both associated with homeostasis of lymphocyte populations, with IL2 being particularly implicated in the function of regulatory $\mathrm{T}$ cells $\left(\mathrm{T}_{\mathrm{reg}}\right)$. Additional intriguing candidate gene associations that need further replication include KIAAO35O, a gene of unknown function expressed in immune cells, and $C_{5} 8$, encoding a ligand for the activation receptor $\mathrm{CD} 2$ on $\mathrm{T}$ cells.

Both shared and unique genes are linked with individual autoimmune diseases. Linda Wicker (University of Cambridge, UK) summarized current finding on genetic linkages with type 1 diabetes. Here again, the HLA class II region is most highly associated with disease predisposition and, as in MS, the $I L 2 R A$ gene is linked with type 1 diabetes. The insulin locus is implicated as a gene uniquely linked with type 1 diabetes. The R620W allele of protein tyrosine phosphatase 22 (PTPN22) is associated with type-1 diabetes. PTPN22 binds to CSK kinase and this complex negatively regulates T-cell signaling. The R620W mutation results in disrupted binding of PTPN22 to CSK kinase, suggesting that this polymorphism may result in alterations in T-cell activation to autoimmune stimuli. R620W is also associated with RA, but not with MS. In contrast, CTLA4, another negative regulator of T-cell activation, is associated with type 1 diabetes, but not with MS or RA. The fact that distinct immune-function genes are linked with specific diseases probably points to differences in the influence of individual immune-response genes in the disease etiology. 


\section{Gene function in animal models of autoimmune disease}

Why are these predisposing genes associated with autoimmunity? For this, we turn to the discussions of immune mechanisms associated with autoimmunity. The analysis of genetic contributions to autoimmune disease susceptibility in animal models is one approach to understanding how underlying genetic changes may contribute to disease. These genes then have to be placed in the context of the immune mechanisms that contribute to autoimmune disease.

The HLA complex is identified as a susceptibility locus in many autoimmune diseases, underscoring the likely requirement for T-cell-dependent adaptive immune responses in autoimmunity. Goverman presented results on the development of experimental autoimmune encephalomyelitis (EAE) in two strains of mice that are congenic except for differences at the MHC class II locus. Both mice made $\mathrm{CD} 4^{+}$ T-cell responses to myelin oligodendrocyte glycoprotein peptides, although the $\mathrm{T}$ cells from each strain recognized distinct immunodominant peptides. This difference in the autoreactive T-cell response resulted in dramatic differences in the course of the disease, with one strain exhibiting classical EAE with ascending paralysis, whereas the other strain had atypical EAE. The current emphasis of these studies is on understanding the pattern of trafficking of these $\mathrm{T}$ cells into the central nervous system, and on the phenotype of the pathogenic cells. As discussed by Steve Miller (Northwestern University, Chicago, USA), activation of pathogenic $\mathrm{T}$ cells in EAE can occur within the central nervous system, and the precise conditions of those priming events can result in distinct disease outcomes. One can extrapolate from these results in animals to considering the effects of small changes in HLA loci on autoimmune disease in humans. Thus, polymorphisms in HLA may not only be permissive for disease, but may also influence disease manifestations.

Genetic mapping studies have also uncovered linkages to immune-function genes in animal models of SLE. Ward Wakeland (University of Texas Southwestern Medical Center, Dallas, USA) reported on the genetics of animal models of SLE. In these studies, allelic regions are isolated from complex resistant and susceptible genetic backgrounds. The SLE1 region was identified on chromosome 1 from the lupus prone strain NZM2410, and is associated with an increased expression of anti-chromatin autoantibodies. Genes encoding members of the SLAM family of receptors are located in this region. The $L y 108$ gene has been implicated in contributing to the loss of B cell tolerance in the susceptible strain. Additional candidate genes in this region include Ly9 and CD84. This region is of particular interest, as a syntenic region in human shows some evidence of linkage in SLE. Toll-like receptors (TLRs) have been implicated in animal models of SLE, as highlighted by Mark Shlomchik (Yale University, New Haven, USA). In the MRL- lpr mouse model of SLE, deficiency in TLR9 resulted in attenuated auto-antibody responses to chromatin and double-stranded DNA, whereas deficiencies in TLR7 were associated with decreased autoantibody responses to RNA antigens. However, deficiency in TLR9 was associated with increased clinical signs of lupus nephritis in these mice, whereas deficiency in TLR7 was protective. Thus, genetic alterations in specific TLR receptors can affect distinct disease parameters. Ann Marshak-Rothstein (Boston University, USA) presented recent results identifying a role for the receptor for advanced glycation end products (RAGE) and its ligand HMGB1 (amphoterin) in the activation of autoreactive B cells in SLE models. These studies suggest that innate immune pathways may contribute to SLE pathology.

\section{Co-stimulation and autoimmunity}

The CD28 gene family and their B7 family ligands are critical for controlling normal immune responses, and have been implicated in animal models of autoimmune disease. Arlene Sharpe (Harvard Medical School) reported on the current state of knowledge of the $\mathrm{B} 7$ and $\mathrm{CD} 28$ gene families, including an unexpected finding that mouse PD-L1, a B7 family ligand for the PD-1 receptor, can also interact with B7-1 (CD80), another B7 family ligand. Measurements of the avidities of B7.1 and PD-L1, in the form of dimeric fusion proteins with $\mathrm{Fc}$ portion of IgG, suggest that B7.1 binds PDL1 with an avidity intermediate between its avidity for $\mathrm{CD} 28$ and CTLA4. Thus, in considering the effects of the CTLA4 polymorphisms in autoimmunity, one must integrate this shared ligand paradigm for B7.1 binding to CD28, CTLA4, and now PD-L1. The costimulatory receptor ICOS has been shown to be important for IL21 production by follicular T helper cells, as discussed by Joe Craft (Yale University), providing a possible biological rationale for a linkage with antibody dependent autoimmune disease. IL21 has also been implicated in animal models of type 1 diabetes, RA and SLE.

Another gene possibly associated with type 1 diabetes in the studies discussed by Wicker is CD226, which encodes an immunoglobulin superfamily member that has been identified as a co-stimulatory receptor. Ligands for this protein are the immunoglobulin superfamily members PVR and CD112 (Nectin 2). Steve Levin (ZymoGenetics, Seattle, USA) reported on a new receptor for PVR and CD112 - Vsig9. His results support a negative regulatory function for this new receptor. Together, these results now link this receptorligand complex with type 1 diabetes.

\section{Regulatory T cells and Th 17 cells: new lineages of $T$ cells}

A key theme of the conference was the balance between regulatory $\mathrm{T}$ cells $\left(\mathrm{T}_{\mathrm{reg}}\right)$ and a newly identified lineage of effector CD4 T cells called Th17 cells. Th17 cells are characterized by the production of the cytokines IL17A, IL17F and 
IL22, and have been implicated as pathogenic effector cells in several models of autoimmune disease.

In contrast, $\mathrm{T}_{\text {reg }}$ cells are thought to be critical for the maintenance of tolerance and for limiting immune responses. The importance of $\mathrm{T}_{\text {reg }}$ in humans is underscored by identification of mutations in $\mathrm{FoxP}_{3}$, a transcription factor required for $\mathrm{T}_{\text {reg }}$ function, in patients with the autoimmune disease IPEX (immunodysregulation, polyendocrinopathy and enteropathy). Deficiency in $\mathrm{FoxP}_{3}$ results in an autoimmune phenotype that is lethal in childhood if untreated. In numerous mouse models, $\mathrm{T}_{\text {reg }}$ cells have been found to modulate susceptibility to autoimmune disease as well as disease course.

Both $\mathrm{T}_{\text {reg }}$ and Th17 cells require transforming growth factor beta (TGF $\beta$ ) for their differentiation, but the presence of IL6 biases towards development of a Th17 response. Richard Flavell (Yale University) presented data from TGF $\beta$ conditional knockout mice, demonstrating that TGF $\beta$ made by $\mathrm{T}$ cells is critical for the development of Th17 cells. In contrast, T-cell derived TGF $\beta$ is not required for development of $\mathrm{T}_{\text {reg }}$, implying that other sources of TGF $\beta$ can suffice. Vijay Kuchroo (Harvard Medical School) discussed new results showing that IL21 can also contribute to the development of Th17 cells, providing one possible rationale for the linkage of the IL21 locus to autoimmunity in animal models.

Rachel Caspi (National Eye Institute, NIH, Bethesda, USA) presented results showing that Th17 cells contribute to the pathology of experimental autoimmune uveitis (EAU), an autoimmune inflammation of the eye, in mice. Interestingly, although antibodies to IL17A block EAU, IL17A-deficient mice appear to be susceptible to EAU. Thus, multiple effector molecules must contribute to this disease, and in the absence of IL17A, a distinct autoimmune profile must emerge. This illustrates the importance of genetic context in querying the contribution of a particular mediator.

It is interesting that CD25, the high-affinity receptor for IL2, is linked to multiple human autoimmune diseases. CD25 is expressed on $\mathrm{T}_{\text {reg }}$ cells, and IL2 is required for $\mathrm{T}_{\mathrm{reg}}$ function. As outlined by Calliope Dendrou (University of Cambridge), current efforts are directed towards understanding how CD25 polymorphisms may influence $\mathrm{T}_{\mathrm{reg}}$ and $\mathrm{T}$-cell effector functions. In preliminary studies, normal human volunteers bearing susceptibility alleles at CD25 were found to express lower levels of CD25 on the surface of their Treg cells. The CD25 susceptibility allele was also correlated with lower levels of soluble CD25 in serum. Functional studies on Treg will be required to evaluate how this may influence $\mathrm{T}$ cell responses.

Our ability to intervene in autoimmune diseases will be greatly enhanced by an understanding of the roles of these genetic pathways in normal immune responses. With new tools, such as the ability to image responding immune cells using intravital microscopy, as elegantly presented by Ron Germain (National Institute of Allergy and Infectious Diseases, $\mathrm{NIH}$, Bethesda, USA) and Uli von Andrian (Harvard Medical School), we can now begin to visualize immune responses in vivo. Ron Germain highlighted his recent findings examining migration of $\mathrm{T}$ and $\mathrm{B}$ cells in lymph nodes in vivo. These studies are done by labeling cell populations with fluorescent tags that can be visualized directly in lymph nodes using confocal and multiphoton intravital microscopy. $\mathrm{T}$ cells were visualized migrating along fibroblastic reticular networks, whereas follicular B cells migrated along a distinct dendritic follicular network. These studies are now being extended to examine the migration of antigen-specific CD4 and CD8 T cells and B cells in response to antigen associated with dendritic cells. This work allows a more accurate picture of how an immune response occurs in space and time within the lymphoid tissues.

Using similar methodologies, Uli von Andrian has begun to examine cellular interactions in the context of viral infection. Injection of fluorescently labeled VSV virus in mice resulted in capture of the virus by a distinct population of $\mathrm{CD} 169+$ macrophages in the draining the lymph nodes. These cells then presented antigen to B cells within the nodes, allowing for initiation of an anti-viral immune response.

As the in vivo architecture of the immune response becomes defined, one can predict that genetic variation will also impact the spatial and kinetic aspects of this response. These techniques will allow acquisition of new information about how immune responses are regulated by specific genes.

What are the implications of finding that predisposing genes are immune-function genes? One is that multiple small changes in the function of certain immune proteins can lead to profound changes in our response to immune stimuli. Each of the polymorphisms detected could result in a functional, but slightly different, immune response. Under the right conditions, and with the right mixture of complex genes, these small changes are enough to tip the balance. Another indication from these results is that immune stimuli are implicated in the initiation of autoimmune diseases, supporting the hypothesis that infectious diseases or other inflammatory events may be temporally linked to the initiation of autoimmune disease.

Why have we retained these predisposing genes in the general population? Profound changes are likely to be selected against, as survival from infectious disease would be compromised. Rather, these smaller changes may have been selected to allow greater survival in endemic diseases such as tuberculosis or malaria. Understanding the pathological processes that drive autoimmune disease may allow us to target therapy more precisely and allow us to identify those patients who can respond to particular therapeutic options, without compromising their ability to respond to infectious disease. 